Europe's Journal of Psychology, 6(3), pp. 71-93

www.ejop.org

\title{
Perceiv ed Parental Warmth and Rejection in Childhood as Predictors of Humor Styles and Subjective Happiness
}

\author{
Shahe S. Kazarian \\ American University of Beirut \\ Lamia Moghnie \\ Univ ersity of Michigan \\ Rod A. Martin \\ Univ ersity of Western Ontario
}

\begin{abstract}
This research examined maternal and paternal warmth (acceptance) and rejection (hostility and aggression, indifference/neglect, and undifferentiated rejection), as remembered by young adults, in relation to humor styles and subjective happiness. A total of 283 Lebanese college students completed the Arabic versions of the Adult Parental Acceptance-Rejection Questionnaire for Mother and Father, the Humor Styles Questionnaire, and the Subjective Happiness Scale. As predicted, parental warmth correlated positively and parental overall rejection and specific rejection scores correlated negatively with subjective happiness ratings. Parental warmth tended to correlate positively with use of adaptive humor styles, and negatively with use of maladaptive humor styles, while parental rejection tended to correlate positively with use of maladaptive humor styles and negatively with use of adaptive humor styles. In addition, self-enhancing humor mediated the relationships between parental warmth and rejection and subjective happiness. Overall, the findings are consistent with the view that parental warmth and rejection might contribute to the development of particular styles of humor, which in turn may contribute to later happiness and well-being.
\end{abstract}

Keywords - humor styles, parental acceptance/rejection, subjective happiness. 
Parental acceptance and rejection theory (PARTheory) is a pancultural socialization theory that focuses on the warmth and rejection dimensions of parenting and their consequences on the behavioral, cognitive, and emotional development of children and personality functioning of adults in different cultures and populations (Khaleque, Rohner, Riaz, Laukkala \& Sadeque, 2007; Rohner, Khaleque \& Cournoyer, 2009). Warm and accepting parents tend to show physical and verbal love and affection toward their children, thus inducing in them feelings of being loved and accepted. Rejecting parents, on the other hand, tend to dislike, disapprove of, or resent their children, thus invoking in them feelings of rejection or being unloved and unwanted. The Perceived Parental Acceptance/Rejection Questionnaire (PARQ; Rohner \& Khaleque, 2005a; Rohner, Saavedra \& Granum, 1978a), which comes in three versions (child, adult, and parent), is the most widely used measure of parental warmth and rejection. It assesses three forms of rejection: (1) hostility and aggression, (2) indifference and neglect, and (3) undifferentiated rejection, which refers to the belief that one is not cared about or loved by parents, even in the absence of clear parental behaviors of neglect or aggression.

\section{PART Personality Subtheory}

The personality subtheory of PARTheory attempts to identify and predict the consequences of perceived parental acceptance and rejection on the behavioral and personality dispositions of children and their personality functioning in adulthood (Rohner et al, 2009), as measured by the Personality Adjustment Questionnaire (PAQ; Rohner \& Khaleque, 2005b; Rohner, Saavedra, \& Granum, 1978b). The PAQ measures seven personality traits or dispositions that collectively reflect psychological maladjustment: hostility/aggression; dependence; negative self-esteem; negative self-adequacy; emotional unresponsiveness; emotional instability; and negative worldview. Studies that have examined the relation between parental acceptance and rejection and psychological adjustment among children and adults have invariably found that rejected individuals, regardless of their gender, culture, race, or socioeconomic status, show more negative personality traits and overall psychologic al maladjustment than do accepted children (Rohner \& Khaleque, 2002; Rohner, 2004, 2005; Rohner et al., 2009). Similarly, cross-cultural and intracultural studies have provided evidence of worldwide correlations between parental rejection and other mental health problems including anxiety and insecurity, depression, borderline personality disorder, substance use, and behavioral problems such as conduct disorder and delinquency (Rohner \& Britner, 2002; Rohner et al., 2009). 
PARTheory Personality Subtheory: Expansion and Extension

Researchers in this topic area have focused particularly on the universality of the association between parental rejection and specific forms of psychological maladjustment, and on the implications of this association for the enhancement of the welfare of humanity in the global village (Rohner, 2004). Nevertheless, the main assumption of the theory (parental rejection has negative effects on psychological adjustment and personality functioning of both children and adults) continues to stimulate confirmation of the postulate in different countries, particularly the culturally rich but politically volatile Arab Middle East. Thus far, several studies in the region of the Arab Middle East have supported the association between parental warmth and psychological adjustment: Bahraini youth (Al-Falaij, nd), students in the Kingdom of Saudi Arabia (Aljasir, 2006; Alzahrani, 2009; Zidan, nd), Jordanian adolescents (Dwairy, 2010), Egyptian and Yemeni children (Asker, 1996), Egyptian college students (Salama, 1986), Qatari children (Khaliefa, 2004), and married adults in Kuw ait (Parmar, Ibrahim, \& Rohner, 2008).

Nevertheless, previous research on the consequences of the parental "acceptancerejection syndrome" has been in the main focused on the constellation of the seven personality dispositions as assessed by the PAQ. The advent of positive psychology which has widened the scope of psychology to the science of happiness and positive individual traits (Seligman, 2000), and the recent resurgence of interest in sense of humor as a personality variable (Martin, 2007) have allowed exploration of parenting behaviors in relation to positive personality dispositions and somatic, psychological, and social outcomes beyond the "magnificent seven" on which PARTheory's personality subtheory has been grounded. As such, in the present study, the consequences of parental warmth on sense of humor are examined. Consideration of humor styles in the context of the universalist "acceptancerejection syndrome" is particularly relevant in view of the paucity of research on the developmental trajectories of humorous personality traits ge nerally (Martin; Vernon, Martin, Schermer, \& Mackie, 2008), and the absence of any study on the association of parental warmth and rejection with humor styles. Additionally, the recent reformulation of sense of humor as a personality disposition with both adaptive and maladaptive dimensions provides a conceptual framework for exploring the differential socialization of humor styles and evaluation of their beneficial and detrimental effects on subjective happiness, in a similar way to those studies that have related humor styles to physical, psychological, and social well-being (Kuiper \& Harris, 2009; Martin, 2001, 2007). 


\section{The Present Study}

In the present study, the assumption of PARTheory that parental warmth and rejection in childhood are associated with subjective well-being in adulthood was tested in the Lebanese context, using a measure of subjective happiness to assess well-being. To our knowledge the consequences of parental acceptance-rejection, as conceptualized by PARTheory, on the cognitive, emotional, and behavioral function of young Lebanese adults is not known. It was important to demonstrate empirically that predictions from the PARTheory apply to young adults in Lebanese culture. Two previous studies in the Arab Middle East have used the Adult PARQ to examine maternal acceptance in Egypt and maternal and paternal acceptance in Kuwait in relation to adult psychological adjustment but not subjective happiness (Parmar et al., 2008; Salama, 1986).

A second aim of the study was to use the Arabic Humor Styles Questionnaire to explore the relation between parental warmth and rejection and two potentially adaptive (Affiliative and Self-Enhancing) and two potentially maladaptive (Aggressive and Self-Defeating) humor styles (Martin, Puhlik-Doris, Larsen, Gray, \& Weir, 2003). To our knowledge, humor styles have not been examined before in relation to the "parental acceptance-rejection syndrome." Such a study extends the constellation of the personality dispositions measured by the PAQ that have been universally associated with parenting (Rohner \& Khaleque, 2002) to the sense of humor domain. Affiliative humor involves the use of humor to enhance one's relationships with others by saying funny things, telling jokes, and engaging in spontaneous witty banter to amuse others, to facilitate relationships, and to reduce interpersonal tensions. Self-Enhancing humor involves the use of humor to regulate emotions and cope with stress through a humorous outlook on life, frequently being amused by the incongruities of life, and maintaining a humorous perspective even in the face of stress or adversity. Aggressive humor involves the use of humor to enhance the self at the expense of others, by means of sarcasm, teasing, ridicule, derision, "put-down" humor, or disparagement humor. Self-Defeating humor involves the use of humor to enhance relationships at the expense of the self by means of excessiv ely self-disparaging humor, amusing others by doing or saying funny things at one's own expense as a way of ingratiating oneself to others or gaining approval, and using humor as a form of defensive denial or avoidance.

A considerable amount of research has demonstrated that these four humor styles are differentially related to particular aspects of psychosocial health and well-being (see Martin, 2007 for a review of this research). In particular, Self-enhancing humor 
tends to be associated with lower levels of depression and anxiety and higher selfesteem and general emotional well-being. Affiliative humor, while somewhat more weakly positively correlated with emotional well-being, tends to be particularly associated with satisfactory interpersonal relationships. Aggressive humor, although generally unrelated to emotional well-being, tends to be negatively correlated with relationship satisfaction. Finally, Self-defeating humor has been found to correlate positively with depression and anxiety, and negatively with self-esteem and emotional well-being in North American (Martin et al., 2003), European (Saroglou \& Scariot, 2002), and Chinese samples (Chen \& Martin, 2007). However, in past research with Middle East samples, Self-defeating humor has generally been found to be unrelated to measures of emotional well-being (Kazarian \& Martin, 2004; Kazarian \& Martin, 2006; Taher, Kazarian, \& Martin, 2008), suggesting that this selfdisparaging and ingratiating form of humor may not be as clearly detrimental to well-being in the Middle Eastern cultural context as it appears to be in some other cultures. Based on this past research, we expected that in this Arabic-speaking sample, Affiliative and Self-enhancing humor would be positively correlated with subjective happiness whereas Self-defeating and Aggressive humor would be unrelated to this variable. Such a finding would provide further support to the distinction between beneficial and detrimental humor styles.

Furthermore, and consistent with the expanded PARTheory's personality subtheory, we expected a link between parental warmth and rejection in childhood and adult use of adaptive and maladaptive humor. More specifically, we hypothesized that remembered parental warmth and acceptance in childhood would correlate positively with Affiliative and Self-Enhancing humor styles and negatively with Aggressive and Self-Defeating humor styles. Similarly, we expected overall parental rejection and the specific forms of rejection to correlate negatively with adaptive humor styles and positively with maladaptive humor styles. Such findings would provide support for the view that parental interactions during childhood may have a lasting effect on personality development (in this case, humor styles).

The third and final aim of this study was to examine the potential me diating effect of humor styles on the relation between recalled parental warmth and rejection in childhood and subjective happiness in young adulthood. We hypothesized that adaptive and maladaptive humor styles may be one pathway by which early experiences of parental warmth and rejection may lead to increased or decreased happiness. For example, individuals who experience greater parental warmth during childhood may be more likely to develop affiliative and self-enhancing styles of humor during the course of development, which in turn might contribute to higher levels of subjective happiness later in life. On the other hand, individuals who 
experience greater parental rejection during childhood may be less likely to develop adaptive styles of humor, which in turn might contribute to lower levels of subjective happiness.

\section{Method}

Participants

A total of 283 Lebanese university students (62.5\% female) participated in the study. The majority of the participants were from the Lebanese University ( $n=193)$, a staterun institution of higher learning, and the remainder from the American University of Beirut, a private educational setting. The mean age $w$ as 20.8 years ( $S D=3.34$, range $=17$ to 44$)$. Reported marital status was $89.6 \%$ single, $7.9 \%$ married, and $2.5 \%$ other.

\section{Measures}

Arabic Adult Parental Acceptance-Rejection Questionnaire for Mother and Father (PARQ). The Arabic Adult PARQ for Mother and Father (Rohner \& Khaleque, 2005a; Rohner et al., 1978a) are two self-report measures of adults' perceptions of maternal and paternal acceptance and rejection when they were about seven to 12 years of age. The Mother and Father versions, each comprising 60 items, differ only in which parent they refer to. Each measure provides an overall rating and scores on four parental behavioral domains: Warmth (20 items; e.g., "Said nice things about me), Hostility/Aggression (15 items; e.g., "Ridiculed and made fun of me"), Indifference/Neglect (15 items; e.g., "Paid no attention when I asked for help"), and Undifferentiated Rejection (10 items; e.g., "Did not really love me"). Respondents rate separately their mothers' and fathers' warmth and rejection using a 4-point Likert scale ( 4 = almost always true, 1 = almost never true), higher scores reflecting higher overall rejection, Hostility/Aggression, Indifference/Neglect, Undifferentiated Rejection, and Warmth. Overall Adult PARQ rejection scores range from 60-240, with scores below the midpoint of 150 indicating recollections of more acceptance than rejection in childhood, and scores above the midpoint indicating more recalled rejection than acceptance. The Mother and Father versions of the PARQ have been $\checkmark$ alidated cross-culturally with robust reliability and validity findings including internal consistencies exceeding .70 for the overall scores and the four domains (Khaleque \& Rohner, 2002; Rohner \& Couroyer, 1994). Similarly, the Arabic PARQ has shown appropriate internal consistencies (Parmar et al., 2008; Salama, 1986). In the present study, the internal consistencies (Cronbach alphas) of the Arabic Adult PARQ ratings of Mother were .74 for overall ratings, .90 for warmth, .87 for Hostility, .83 for 
Indifference/Neglect, and .77 for Undifferentiated Rejection, while those of Father were .78 for overall ratings, .93 for warmth, .92 for Hostility, .83 for Indifference/Neglect, and .84 for Undifferentiated Rejection.

Arabic Humor Styles Questionnaire (HSQ). The Arabic HSQ (Martin et al., 2003 ; Taher et al., 2008) is a 32-item measure comprising four 8-item scales assessing different styles of humor: Affiliative (e.g., "I laugh and joke a lot with my friends"); SelfEnhancing (e.g., "My humorous outlook on life keeps me from getting overly upset or depressed about things"); Aggressive (e.g., "If someone makes a mistake, I will often tease them about it"); and Self-Defeating humor (e.g., "I often try to make people like or accept me more by saying something funny about my own weaknesses, blunders, or faults"). Respondents indicate the degree to which they agree with each item using a 7-point Likert scale. Martin et al. (2003) reported internal consistencies for the four scales in the range of .77 to .81 . In the present study, the internal consistencies of the Arabic HSQ were .66 for Affiliative, .74 for Self Enhancing, .59 for Aggressive and .69 for Self Defeating humor, findings comparable to those reported by Taher et al.

The Arabic Subjective Happiness Scale (SHS). The SHS (Lyubomirsky \& Lepper, 1999) is a 4-item global measure of the degree to which one is a happy or an unhappy person. Respondents are asked to respond to the items on a scale of 1 (not a very happy person) to 7 (very happy person), higher scores reflecting higher subjective happiness. Lyubomirsky and Lepper reported internal consistencies ranging from .79 to .94 for the original version of the SHS, and Swami (2008) reported an internal consistency of .93 for a Malay version. For the present study, the original version of the SHS was translated into Arabic by a professional translator, and the Arabic version was then translated back to English by another professional translator, independent of the first translator. The two English versions were then compared and differences reconciled. Care was taken to ensure equiv alence rather than literacy in translation. In the present study, the internal consistency of the Arabic SHS was .75.

Procedure

Participants completed the measures in group testing sessions. All measures were administered in the Arabic language. The three measures were administered to participants in randomized order to control for potential order effects. 
Results

Descriptive Statistics and Gender Differences

The means and standard deviations for all the measures for the total sample and for females and males separately are presented in Table 1. The mean overall rejection

Table 1: Means and standard deviations of all the measures for the total sample $(N=283)$, females $(n=177)$, and males $(n=101)$, and significance of t-tests comparing means for males and females.

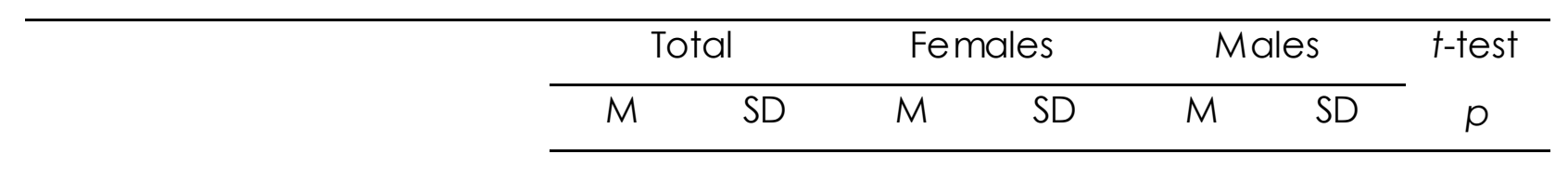

Humor Styles

Affiliative

Self-Enhancing

Aggressive

Self-Defeating

$\begin{array}{lllllll}41.7 & 8.48 & 42.0 & 8.154 & 40.7 & 9.06 & \mathrm{~ns} \\ 35.8 & 9.36 & 35.6 & 9.49 & 36.3 & 9.15 & \mathrm{~ns} \\ 24.3 & 8.30 & 23.3 & 7.86 & 26.0 & 8.68 & .009 \\ 21.7 & 8.54 & 20.5 & 8.15 & 23.7 & 8.76 & .003\end{array}$

Ratings of Mothers

Warmth

Hostility/Aggression

Indifference/Neglect

$\begin{array}{lllllll}68.4 & 10.18 & 69.0 & 9.64 & 67.1 & 11.08 & \text { ns }\end{array}$

Undifferentiated

27.8

23.

7.52

$27.3 \quad 7.88$

$28.4 \quad 6.94 \quad \mathrm{~ns}$

17.8

6.26

22.6

6.04

24.0

$6.62 n s$

Rejection

Overall Rejection

$\begin{array}{lllllll}96.0 & 23.77 & 94.4 & 24.70 & 98.6 & 22.26 & n s\end{array}$

Ratings of Fathers

Warmth

$\begin{array}{lllllll}65.2 & 12.09 & 67.1 & 11.03 & 62.0 & 13.20 & .001\end{array}$

Hostility/Aggression

$\begin{array}{lllllll}27.0 & 9.54 & 24.6 & 8.55 & 31.3 & 9.87 & .001\end{array}$

Indifference/Neglect

$\begin{array}{lllllll}27.8 & 7.57 & 26.1 & 6.82 & 30.7 & 8.09 & .001\end{array}$

Undifferentiated

17.9

4.81

$17.9 \quad 5.06$

17.6

$4.47 \quad n s$

Rejection

Overall Rejection

$\begin{array}{lllllll}107.4 & 30.86 & 100.5 & 27.42 & 120.1 & 33.41 & .001\end{array}$

Subjective Happiness

$4.7 \quad 1.17$

4.7

1.18

4.9

1.17

ns 
ratings of Lebanese college youth of their mothers and fathers were below the midpoint of 150. These findings are similar to those reported by Parmar et al. (2008) for Kuwaiti adults, and suggest that as a group Lebanese college youth remembered their mothers and fathers in childhood as substantially warm, loving and accepting. As can be seen in Table 1, Lebanese males and females were comparable in their ratings of their mothers on warmth and the specific forms of rejection. Males, however, rated their fathers significantly lower on warmth $(t(261)=3.32, p<.001)$, and higher on overall rejection $(t(260)=5.13, p<.001)$ and the specific forms of rejection (Hostility/Aggression $(t(261)=5.70, p<.001)$, Indifference/Neglect $(\dagger(261)=4.91, p<.001)$, and Undifferentiated Rejection $(t(261)=4.38, p<.001))$ than did their female counterparts. This pattern of sex differences is similar to that reported by Dwairy (2010) but at odds with findings reported by others (Cournoyer, Sethi, \& Cordero, 2005; Kim \& Rohner, 2003). While no firm conclusions can be drawn about possible sex differences in responses to the mother and father versions of the $P A R Q$, our findings may be unique in reflecting the sex-specific Lebanese cultural image of the "strict father and benevolent mother" (Kim \& Rohner).

With regard to subjective happiness, the mean score of 4.7 for this sample was similar to means reported for US and Russian college students (Lyubomirsky \& Lepper, 1999), and suggests that Lebanese youth in this group construed themselves as having a generally happy disposition. Subjective happiness scores did not differ between males and females, a finding which is also consistent with previous research (Lyubomirsky \& Lepper; Swami, 2007).

With regard to humor styles, mean scores were highest for Affiliative humor followed by Self-Enhancing, Aggressive, and Self-Defeating humor, findings that are comparable to those reported by Taher et al. (2008). With regard to sex differences, males and females reported comparable use of Affiliative and Self-Enhancing humor, but males reported significantly more use of Aggressive $(t(270)=2.62$, $p<$ .009) and Self-Defeating humor $(t(270)=2.98, p<.003)$ than did females. These gender differences are consistent with those found previously in North American and Lebanese samples (Kazarian \& Martin, 2004; Martin et al., 2003).

Perceived Parental Acceptance/Rejection and Subjective Happiness

Table 2 shows the correlations between PARQ parental acceptance-rejection scores and the measures of humor styles and subjective happiness. As postulated, higher scores on maternal and paternal overall rejection and specific rejection ratings were significantly associated with lower reports of subjective happiness. On the other 
hand, as expected, maternal and paternal warmth were significantly associated with higher reports of subjective happiness. Taken together, the findings with subjective happiness support the PARTheory postulate that parental acceptance and rejection are likely predictors of emotional consequences in adulthood.

Humor Styles and Subjective Happiness

As can be seen in Table 2, Affiliative and Self-Enhancing humor, but not Aggressive and Self-Defeating humor, were significantly associated with higher reports of subjective happiness. The lack of association between Self-defeating humor and well-being is consistent with previous research in Middle East samples (Kazarian \& Martin, 2004; Kazarian \& Martin, 2006; Taher, Kazarian, \& Martin, 2008). The differential correlation of adaptive and maladaptive humor styles with subjective happiness supports the conceptual distinction between beneficial and non-beneficial humor styles, as postulated by Martin et al. (2003).

Table 2: Inter-correlations of Arabic Humor Styles Questionnaire, Subjective Happiness, and PARQ Ratings of Mother and Father

\begin{tabular}{lccccc}
\hline & & $\begin{array}{c}\text { Self- } \\
\text { Enhancing }\end{array}$ & Aggressive & $\begin{array}{c}\text { Self- } \\
\text { Defeating }\end{array}$ & $\begin{array}{c}\text { Subjective } \\
\text { Happiness }\end{array}$ \\
\cline { 2 - 6 } Subjective Happiness & $23^{* *}$ & $.37^{* *}$ & -.04 & .01 & -- \\
Ratings of Mothers & & & & & \\
Warmth & $.18^{* *}$ & $.30^{* *}$ & $-.14^{*}$ & $-.16^{* *}$ & $.21^{* *}$ \\
Hostility/Aggression & -.04 & $-.18^{* *}$ & $.21^{* *}$ & $.17^{* *}$ & $-.19^{* *}$ \\
Indifference/Neglect & $-.14^{*}$ & $-.15^{* *}$ & $.20^{* *}$ & $.17^{* *}$ & $-.22^{* *}$ \\
Undiff. Rejection & $-.12^{*}$ & $-.19^{* *}$ & $.20^{* *}$ & $.14^{*}$ & $-.17^{* *}$ \\
Overall Rejection & $-.14^{*}$ & $-.25^{* *}$ & $.21^{* *}$ & $.19^{* *}$ & $-.24^{* *}$ \\
& & & & & \\
Ratings of Fathers & & & & & \\
Warmth & $.17^{* *}$ & $.27^{* *}$ & -.10 & $-.19^{* *}$ & $.25^{* *}$ \\
Hostility/Aggression & $-.12^{*}$ & $-.15^{*}$ & $.14^{*}$ & $.26^{* *}$ & $-.25^{* *}$ \\
Indifference/Neglect & -.12 & $-.15^{*}$ & $.14^{*}$ & $.25^{* *}$ & $-.24^{* *}$ \\
Undiff. Rejection & -.11 & $-.17^{* *}$ & .11 & $.25^{* *}$ & $-.25^{* *}$ \\
Overall Rejection & $-.15^{*}$ & $-.22^{* *}$ & $.14^{*}$ & $.26^{* *}$ & $-.28^{* *}$ \\
\hline
\end{tabular}

Note: $\mathrm{PARQ}=\mathrm{Parental}$ Acceptance-Rejection Questionnaire.

$* p<.05 \quad{ }^{* *} p<.01$ 
Perceived Parental Acceptance/Rejection and Humor Styles

Table 2 also reveals that, as postulated, higher scores on maternal and paternal overall rejection and specific rejection were associated with lower reports of Affiliative and Self-Enhancing humor and higher reports of Aggressive and SelfDefeating humor. Also, scores of maternal and paternal warmth were associated with higher reports of Affiliative and Self-Enhancing humor and lower reports of Aggressive and Self-Defe ating humor. Taken together, these findings provide support for the hypothesis that maternal and paternal warmth during childhood is associated with the development of increased use of adaptive humor styles and decreased use of maladaptive humor styles later in life.

Mediating Effects of Humor Styles

Finally, mediation analyses were conducted to test the hypothesis that humor styles mediate the relationships between parental acceptance/rejection and subjective happiness. These analyses were conducted using SPSS by means of the bootstrap sampling method developed by Preacher and Hayes (2008) for testing the significance of multiple mediators simultaneously. This procedure allows for examination of both the direct effect of the predictor variable on the criterion variable, as well as the indirect effect through the pathway of each of the me diator variables, controlling for all the other mediators. Using sampling with replacement, a large number of samples (1000 in the present study) are drawn from the data set, and path coefficients are calculated for each sample. The mean direct and indirect effects and their confidence intervals (Cls) are then calculated using the estimates from these bootstrap samples. These Cls allow the researcher to determine whether each of the effects is significantly different from zero at a given level of confidence.

In this study, four multiple mediation analyses were conducted separately for maternal and paternal warmth and overall maternal and paternal rejection as the predictor variables and subjective happiness as the criterion variable. In each analysis, only Affiliative and Self-enhancing humor were examined simultaneously as potential mediators, because these two humor styles were the only ones that had significant simple correlations with the overall rejection, warmth, and subjective happiness scores. In order to compare the results across analyses and variables, all variables used in these analyses were standardized $(M=0, S D=1.0)$. Path coefficients can therefore be interpreted in a manner similar to correlation coefficients. 
The results of the mediation analysis for maternal warmth are presented in Figure 1. A significant mediating effect was found for Self-enhancing $(p<.01)$, but not for Affiliative humor. Higher scores on maternal warmth were associated with higher Selfenhancing humor $(a=.32, p<.001)$, which in turn predicted higher happiness scores $(b=.30, p<.001)$. The direct effect of maternal warmth on subjective happiness was not significant $\left(c^{\prime}=.11, n s\right)$, indicating that Self-enhancing humor fully mediates this relationship.

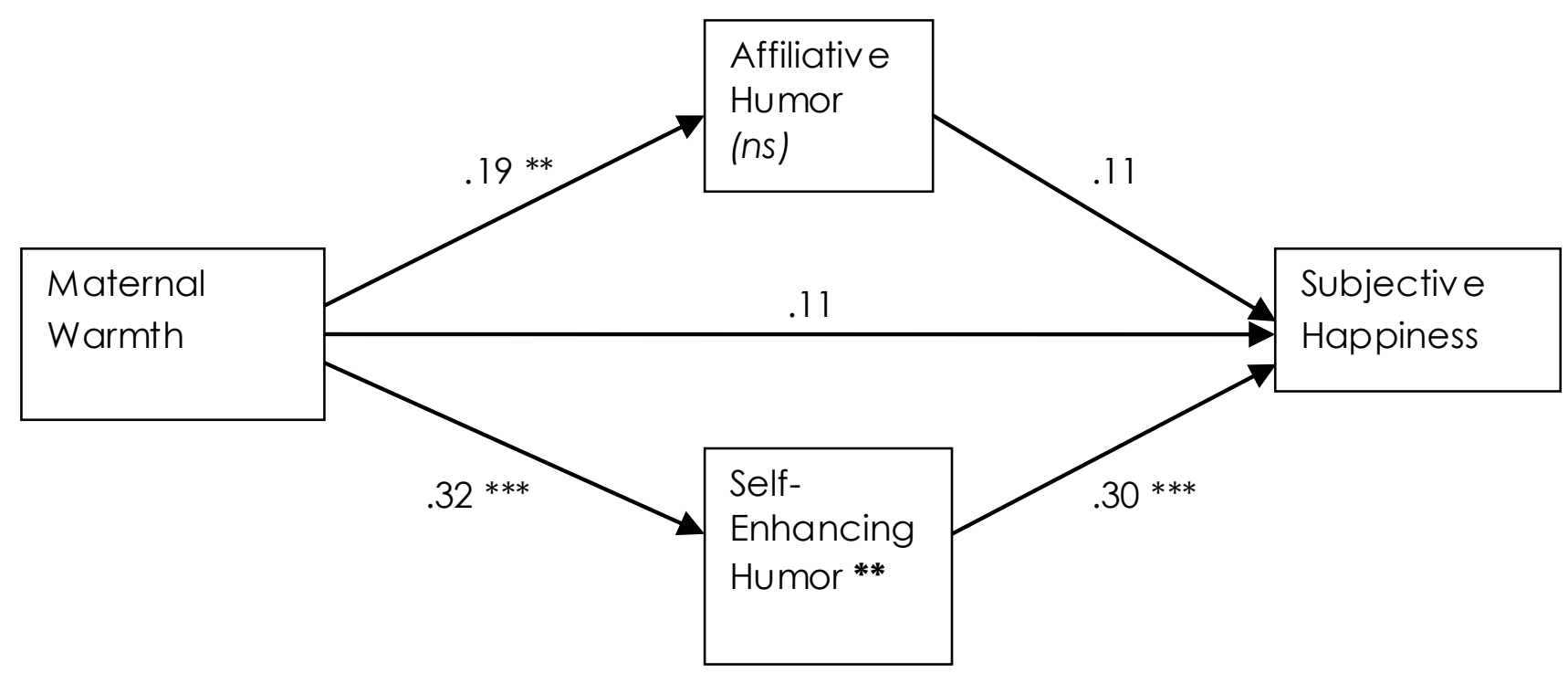

Figure 1: Mediating effects of Humor Styles Questionnaire scales on relationship between Maternal Warmth and Subjective Happiness.

Note: ${ }^{*} p<.05 ;{ }^{* *} p<.01 ;{ }^{* * *} p<.001$

Figure 2 shows the results of the mediation analysis for paternal warmth. Once again, a significant mediating effect was found for Self-enhancing ( $p<.01$ ), but not for Affiliative humor. Higher scores on paternal warmth were associated with higher Selfenhancing humor $(a=.25, p<.001)$, which in turn predicted higher happiness scores $(\mathrm{b}=.33, \mathrm{p}<.001)$. In addition to the indirect effect of paternal warmth on subjective happiness through Self-enhancing humor, a direct effect was also found $\left(c^{\prime}=.17, p\right.$ $<.01$ ), indicating that Self-enhancing humor only partially mediates this relationship. 


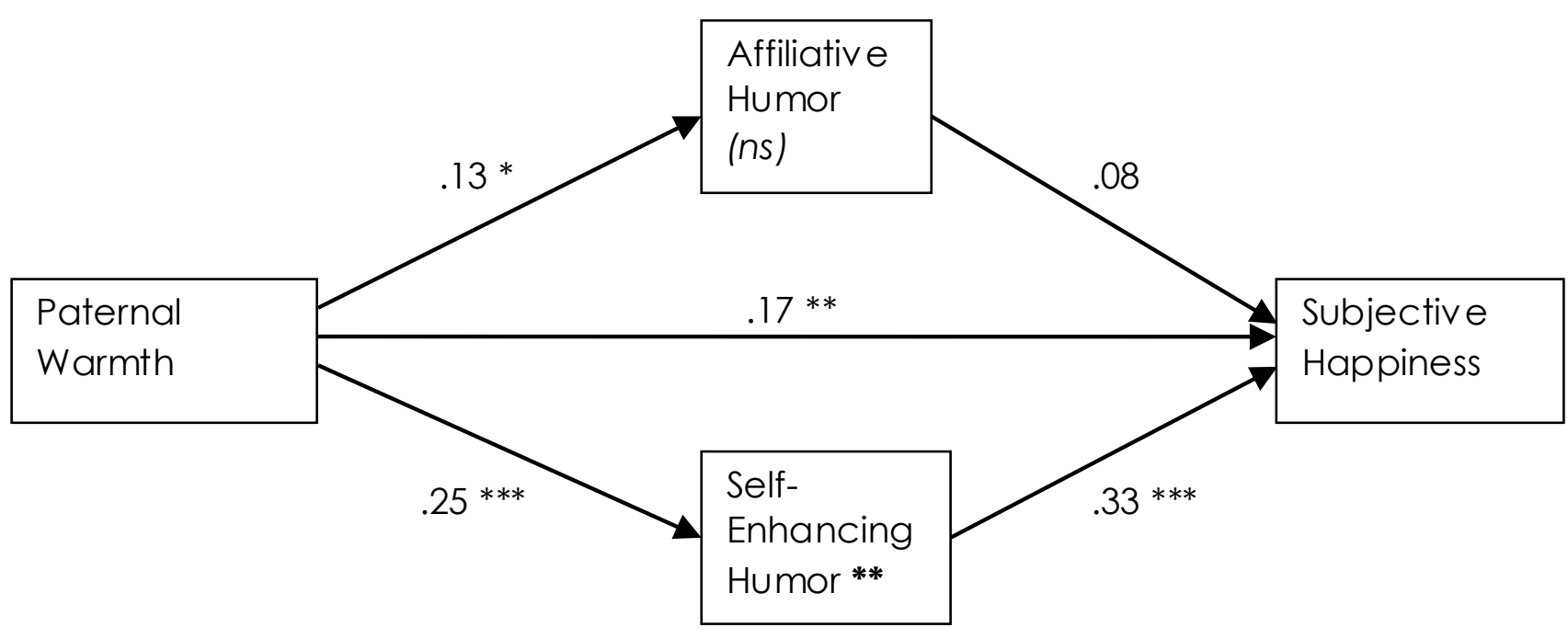

Figure 2: Mediating effects of Humor Styles Questionnaire scales on relationship between Paternal Warmth and Subjective Happiness.

Note: ${ }^{*} \mathrm{p}<.05 ;{ }^{* *} \mathrm{p}<.01 ;{ }^{* * *} \mathrm{p}<.001$

The results of the mediation analysis for overall maternal rejection are presented in Figure 3. Again, a signific ant mediating effect was found only for Self-enhancing ( $p<$ $.01)$ humor. Higher scores on overall maternal rejection were associated with lower Self-enhancing humor $(a=-.25, p<.001)$, which in turn predicted higher happiness scores $(b=.29, p<.001)$. In addition to this indirect effect, a direct effect was also found $\left(c^{\prime}=-.15, p<.05\right)$, indicating that Self-enhancing humor only partially mediates this relationship.

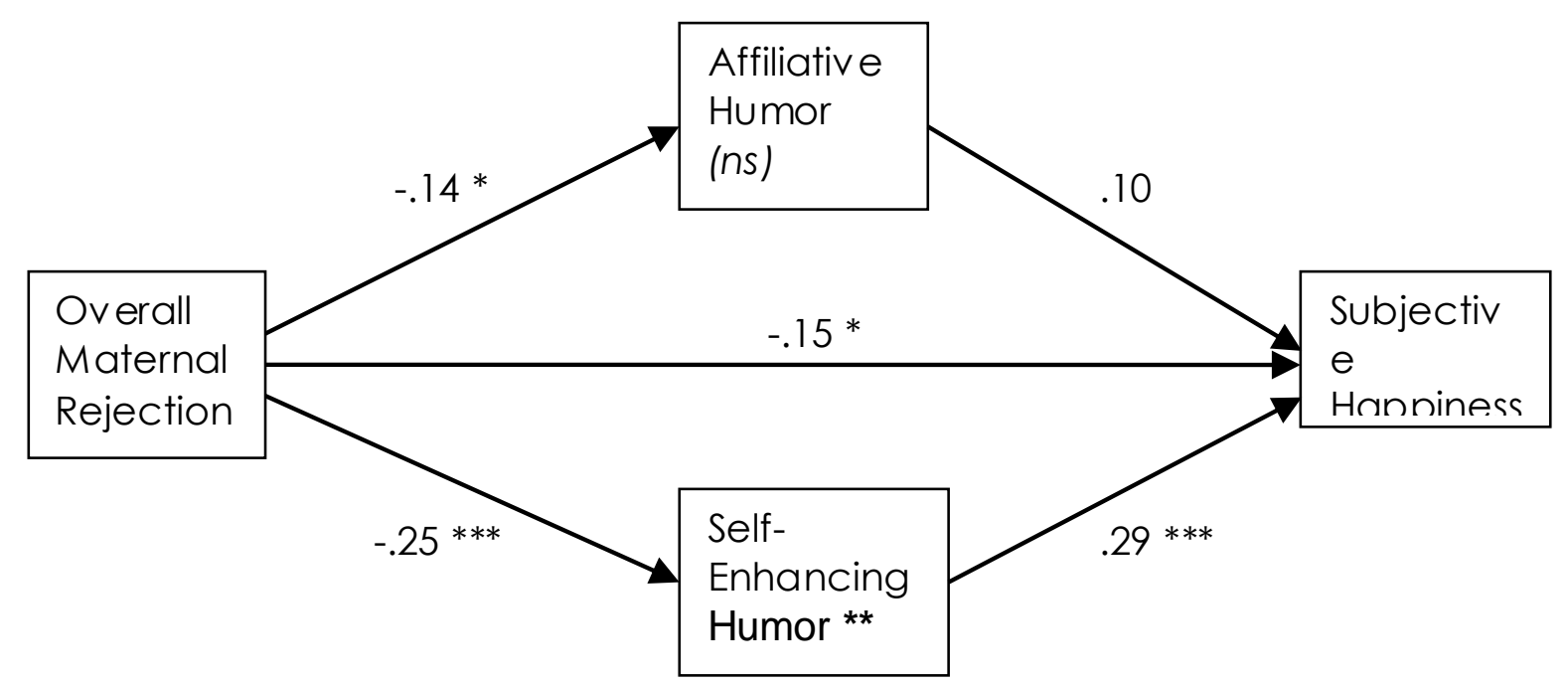

Figure 3: Mediating effects of Humor Styles Questionnaire scales on relationship between Overall Maternal Rejection and Subjective Happiness.

Note: ${ }^{*} p<.05 ;{ }^{* *} p<.01 ;{ }^{* * *} p<.001$ 
Finally, Figure 4 shows the results of the analysis for overall paternal rejection. A significant mediating effect was again found only for Self-enhancing humor $(p<.01)$. Higher scores on overall paternal rejection predicted lower Self-enhancing humor (a $=-.20, p<.01)$, which in turn predicted higher subjective well-being scores $(b=.33, p$ $<.001)$. The direct effect of paternal rejection on subjective happiness was also significant $\left(c^{\prime}=-.22, p<.001\right)$, indicating that Self-enhancing humor only partially mediates this relationship.

\section{Discussion}

This study represents the first application of the Arabic Adult PARQ for Mother and Father and Arabic SHS in Lebanon and the first use of the Arabic HSQ in the context of PARTheory. While PARTheory and its personality subtheory have been focused mainly on measuring and predicting the negative consequences of parental rejection, the present study drew stimulus from positive psychology and the humor styles framework to extend the application of PARTheory to the domains of humor styles and subjective happiness. The psychometric properties of the three Arabic measures used for these purposes were all comparable if not better than those previously reported.

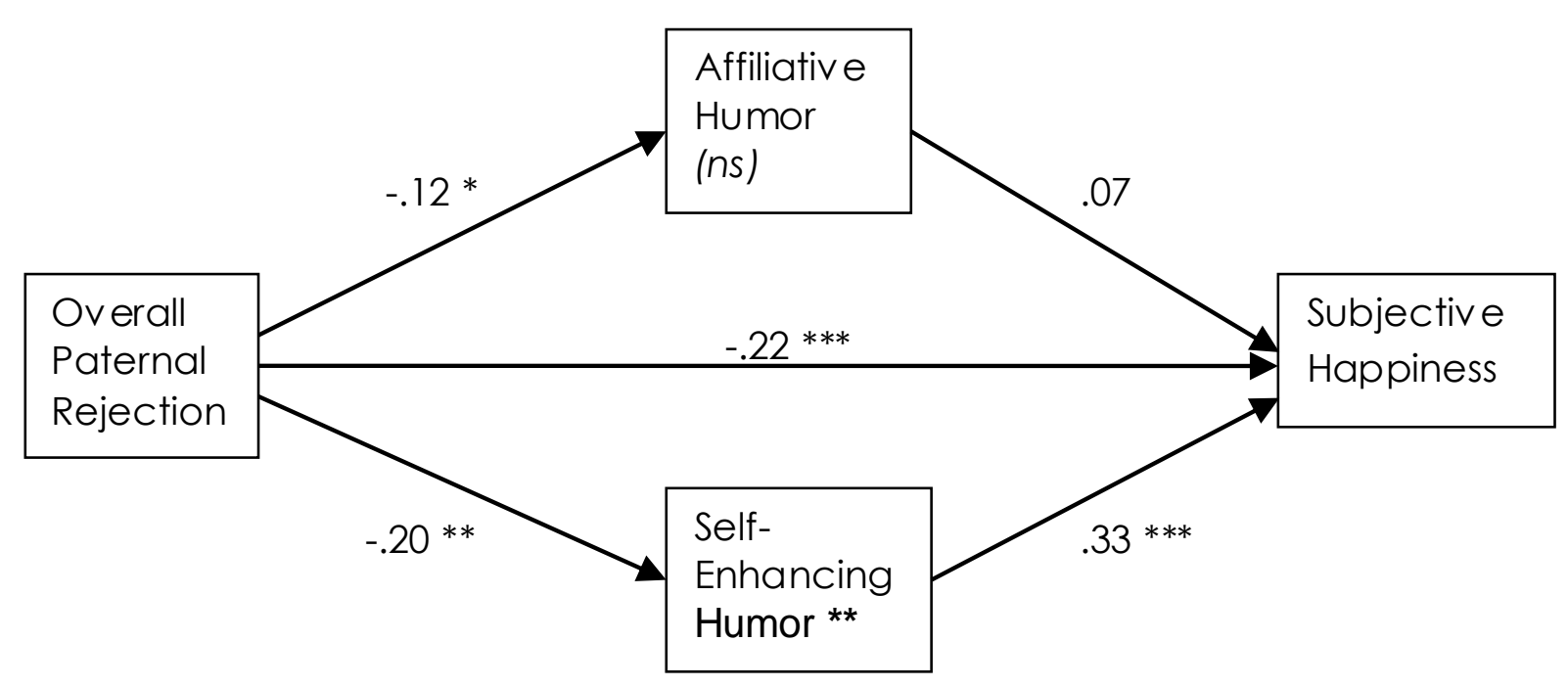

Figure 4: Mediating effects of Humor Styles Questionnaire scales on relationship between Overall Paternal Rejection and Subjective Happiness.

Note: ${ }^{*} \mathrm{p}<.05 ;{ }^{* *} \mathrm{p}<.01 ;{ }^{* * *} \mathrm{p}<.001$ 
Parental Acceptance/Rejection and Subjective Happiness

PARTheory assumes that parental acceptance/rejection in childhood has major consequences on the behavioral, cognitive, and emotional adjustment of children and for the personality functioning of adults. The finding of significant correlations between maternal and paternal warmth and rejection and subjective happiness in the present study not only supports the PARTheory assumption of an association between remembered parenting in childhood and adult functioning but also highlights the positive effect that parental acceptance might have on life-span happiness.

Humor Styles and Subjective Happiness

Martin and colleagues (2003) posit that humor styles are distinguished on the basis of their adaptive and maladaptive functions, Affiliative and Self-Enhancing humor being viewed as potentially beneficial to psychosocial well-being and Aggressive and Self-Defeating humor as potentially detrimental. The finding of significant positive correlations between both of the adaptive humor styles and subjective happiness in the present study is consistent with previous studies on humor styles and psychologic al well-being (Chen \& Martin, 2007; Kazarian \& Martin, 2004; Kazarian \& Martin, 2006; Martin et al., 2003; Saroglou \& Scariot, 2002; Taher et al., 2008). The lack of correlation between Self-defeating humor and subjective happiness is also consistent with previous research in the Middle East (Kazarian \& Martin, 2004; 2006; Taher et al., 2008), although it is inconsistent with the finding of negative correlations between this humor style and well-being variables in other cultures (Chen \& Martin, 2007; Martin et al., 2003; Saroglou \& Scariot, 2002). These results support the assumption of differential associations of the four humor styles with different wellbeing variables as postulated by Martin et al. (2003). They also add further support to the view that the so-called Self-defeating humor style may not be as detrimental to well-being among Middle Eastern cultures. Perhaps a tendency to use humor to disparage oneself, deny one's true feelings, and make others laugh at one's own expense is not viewed as negatively in this culture as in some other cultures.

Parental Acceptance/Rejection and Humor Styles

A main purpose of the present study was to explore the relation between perceived parental acceptance and rejection in childhood and use of adaptive and maladaptive humor in adulthood. Previous research on humor styles has tended to focus particularly on their association with physical and psychological well-being 
and interpersonal relationships. The present study moves this research in the direction of exploring potential childhood origins of these humor styles and particularly the potential contribution of early parenting. Overall, we found that recalled maternal and paternal acceptance were significantly positively correlated with both adaptive humor styles and negatively correlated with the maladaptive humor styles. In addition, overall maternal and paternal rejection correlated positively with the detrimental humor styles and negatively with the beneficial styles. Although many of these associations were relativ ely weak, the strongest correlations were found with self-enhancing humor (in relation to both parental warmth and overall rejection) and with self-defeating humor (particularly in relation to paternal rejection). These findings suggest a possible link between early experiences of parental warmth and rejection and later use of adaptive and maladaptive humor styles. The findings also support predictions from the PARTheory's personality subtheory and extend the subtheory beyond the seven personality disposition domains assessed by the PAQ.

Martin (2007) suggested two possible models of parental influence on humor development: the modeling/reinforcement hypothesis and the stress and coping model. The modeling/reinforcement hypothesis assumes that children develop a good sense of humor in the context of a warm and positive family environment in which the parents provide a role model for humor by laughing and joking frequently and positiv ely reinforce the child's attempts at humor initiation. In contrast, the stress and coping hypothesis suggests that children develop a sense of humor in the context of a cold, rejecting, and uncongenial family environment, in which they learn to use humor to cope with stress and anxiety, relieve interpersonal tension among family members, distract otherwise punitive parents by amusing them, and gain attention and approval from otherwise unsupportive family members.

These two models might account for various aspects of our finding of differential associations between parental warmth and rejection and the four humor styles. In particular, the modeling/reinforcement hypothesis might explain the positive association between parental warmth and self-enhancing and (to a somewhat lesser extent) affiliative humor. Parents who relate to their children in a warm and accepting manner may model and reinforce these positive styles of humor which are then adopted by their children. In contrast, parents who relate to their children in a rejecting, indifferent, or hostile manner likely fail to model and reinforce healthy uses of humor, resulting in a lower likelihood of their children developing selfenhancing and affiliative humor styles. A similar process might account for the positive correlations between parental rejection and aggressive humor, with rejecting parents perhaps modeling and reinforcing aggressive types of humor such as sarcasm and aggressive teasing. On the other hand, the stress and coping model 
might account for the positive associations between overall rejection (particularly paternal) and self-defeating humor. Children who experience these forms of parental rejection may adopt the self-defeating humor style as a method of coping by attempting to make others (including parents) laugh at their own expense, and thereby gain some degree of approval and acceptance in an otherwise uncongenial family environment. These rather speculative hypotheses certainly merit further investigation.

Humor Styles as Mediators of Parenting and Subjective Happiness

Finally, our results also provided some support for the hypothesis that humor styles might mediate the association between early parental acceptance or rejection and later subjective happiness in adulthood. In particular, we found that self-enhancing humor consistently mediated the relationships between both maternal and paternal warmth and rejection and adult subjective happiness. Individuals who recalled higher levels of maternal and paternal warmth reported engaging in more selfenhancing humor, which in turn predicted higher levels of subjective happiness. In contrast, those who recalled higher levels of maternal and paternal rejection reported engaging in lower levels of self-enhancing humor, which in turn was associated with lower levels of subjective happiness. Since self-enhancing humor involves the use of humor to cope with stress, regulate negative emotions, and enhance positive emotions, it is not surprising that this style seems to be particularly important as a mediator in the prediction of happiness. As noted in the previous section, individuals may develop particular styles of humor during childhood in the context of parental warmth and rejection, and these styles of humor may in turn contribute to their level of happiness and other aspects of well-being later in life.

These mediator findings are consistent with other recent research reporting similar mediating effects of humor styles on well-being. Dozois, Martin, and Bieling (2009) found that three of the four humor styles mediated the relationships between several early maladaptive schemas (which are assumed to develop in the context of parent-child relationships) and depression in early adulthood. Similarly, Kuiper and McHale (2009) found that affiliative and self-defeating humor mediated the relationship between positive and negative self-evaluative standards (based on a self-schema model of emotion) and measures of psychological well-being, including depression and social self-esteem. Taken together, these studies suggest that positive and negative humor styles may play an important role in the link between the individual's core self concept, developing in the context of early parenting experiences, and later well-being. Individuals who experience warm and accepting parenting and develop more positive self-standards and adaptive self-schemas may 
consequently develop more beneficial and less detrimental humor styles, which in turn may further enhance social relationships, increasing self-esteem and general well-being. In contrast, early experiences of more rejecting parenting and development of more self-critical and maladaptive core schemas may lead to development of more detrimental and less adaptive humor styles, which in turn result in less positive relationships, increased depression, and lower happiness, self-esteem, and well-being. Overall, then, these findings contribute to a growing body of evidence suggesting that humor styles may be one pathway by which early parenting experiences and self-concept development may influence later wellbeing.

\section{Conclusions, Limitations and Future Research Directions}

To summarize our key findings, maternal and paternal warmth and rejection were differentially associated with use of adaptive and maladaptive humor styles and subjective happiness, and the association between maternal and paternal warmth and rejection and subjective happiness was mediated by self-enhancing humor. Important limitations of this study are the cross-sectional correlational design and the use of retrospective measures of parent-child relationships. Participants' recollections of parental warmth and rejection could have been biased by their current levels of happiness, resulting in significant correlations between these variables despite no actual causal connection. Similarly, rather than humor styles influencing subjective happiness, it is possible that higher levels of subjective happiness cause people to engage in more positive and less negative styles of humor. These possible alternative explanations also apply to the results of our mediator analyses. Given the crosssectional nature of our study, we cannot rule out that, for example, happiness levels or humor styles influence parental warmth or rejection, or that a third variable is responsible for determining both parental behavior and humor styles. Future research using a longitudinal design might be useful in disentangling some of these relationships. While the stress and coping and modeling/reinforcement formulations are possible explanations for the use of humor styles in the context of congenial and non-congenial parental environments, other potential antecedents and mediators need future consideration with child, parent, and non-student adult populations and measures supplementary to the self-report methodology used in the present study. 


\section{References}

Al-Falaij, A. A. (nd). Family conditions, ego development and sociomoral development in juvenile delinquency. Retrieved July 6, 2009, from http://www.bcsr.gov.bh/BCSR/En/ eLibrary/Categories/Thesis/Public/alfalij.htm.

Aljasir, A. A. M. (2006). The relationship between emotional intelligence, self-efficacy and perception of parental acceptance-rejection among male and female students at Umm Al-Qura University [in Arabic]. MA Thesis in partial fulfillment of the requirements for a Master's degree in Developmental Psychology, Umm Al-Qura University, Saudi Arabia.

Alzahrani, A. R. A. (2009). Recognition of acceptance-rejection of parental relationship and the level of ambition among students in the middle stage of Jeddah Governorate [in Arabic]. MA Thesis in partial fulfillment of the requirements for a Master's degree in psychology, Umm Al-Qura University, Saudi Arabia.

Asker, A. E. (1996). Differential cultural study for parental acceptance-rejection between Egyptian and Yemeni children [in Arabic]. Journal of Derasat Nafseya, 6, 275-299.

Chen, G.-H., \& Martin, R. A. (2007). A comparison of humor styles, coping humor, and mental health between Chinese and Canadian university students. Humor: International Journal of Humor Research, 20, 215-234.

Cournoyer, D. E., Sethi, R., \& Cordero, A. (2005). Perceptions of parental acceptancerejection and self-concepts among Ukrainian students. Ethos, 33, 335-346.

Dozois, D. J. A., Martin, R. A., \& Bieling, P. J. (2009). Early maladaptive schemas and adaptive/maladaptive styles of humor. Cognitive Therapy and Research, 33, 585-596.

Dwairy, M. (2010). Parental Acceptance-Rejection: A fourth cross-cultural research on parenting and psychological assessment of children. Journal of Child and Family Studies, 19, 30-35.

Kazarian, S. S., \& Martin, R. A. (2004). Humor styles, personality, and well-being among Lebanese university students. European Journal of Personality, 18, 209-219.

Kazarian, S. S., \& Martin, R. A. (2006). Humor styles, culture-related personality, well-being, and family adjustment among Armenians in Lebanon. Humor: International Journal of Humor Research, 19, 405-423. 
Khaleque, A., \& Rohner, R. P. (2002). Reliability of measures assessing the pancultural association between perceived parental acceptance-rejection and psychological adjustment: A meta-analysis of cross-cultural and intracultural studies. Journal of CrossCultural Research, 33, 87-99.

Khaleque, A., Rohner, R. P., Riaz, M., Laukkala, H., \& Sadeque, S. (2007). Perceived parental acceptance-rejection and psychological adjustment of children: A crosscultural study in Finland, Pakistan, and the United States. Psychological Studies, 52, $114-$ 119.

Khaliefa, B. M. (2004). Children's perceived parental acceptance-rejection and its relation to late childhood problems in primary school in State of Qatar [in Arabic]. Tarbyaet Muaaserat, 68, 173-207.

Kim, S., \& Rohner, R. P. (2003). Perceived parental acceptance and emotional empathy among university students in Korea. Journal of Cross-Cultural Psychology, 34, 723-735.

Kuiper, N. A., \& Harris, A. L. (2009). Humor styles and negative affect as predictors of different components of physical health. Europe's Journal of Psychology. www.ejop.org.

Kuiper, N. A., \& McHale, N. (2009). Humor styles as mediators between self-evaluative standards and psychological well-being. Journal of Psychology, 143, 359-376.

Lyubomirsky, S., \& Lepper, H. S. (1999). A measure of subjective happiness: Preliminary reliability and construct validation. Social Indicator Research, 46, 137-155.

Martin, R. A. (2001). Humor, laughter, and physical health: Methodological issues and research findings. Psychological Bulletin, 127, 504-519.

Martin, R. A. (2007). The psychology of humor: An integrative approach. Burlington, MA: Elsevier Academic Press.

Martin, R. A., Puhlik-Doris, P., Larsen, G., Gray, J., \& Weir, K. (2003). Individual differences in uses of humor and their relation to psychological well-being: Development of the Humor Styles Questionnaire. Journal of Research in Personality, 37, 48-75.

Parmar, P., Ibrahim, M., \& Rohner, R. P. (2008). Relations among perceived spouse acceptance, remembered parental acceptance in childhood, and psychological adjustment among married adults in Kuwait. Cross-Cultural Research: The Journal of Comparative Social Science, 42, 67-76 
Preacher, K. J., \& Hayes, A. F. (2008). Asymptotic and resampling strategies for assessing and comparing indirect effects in multiple mediator models. Behavior Research Methods, 40, 879-891.

Rohner, R. P. (2004). The Parental "Acceptance-Rejection Syndrome": Universal correlates of perceived rejection. American Psychologist, 59, 830-840.

Rohner, R. P. (2005). Parental acceptance-rejection theory studies of intimate adult relationships. Cross-Cultural Research, 42, 5-12.

Rohner, R. P., \& Britner, P. A. (2002). Worldwide mental health correlates of parentalacceptance rejection: Review of cross-cultural and intracultural evidence. Cross-Cultural Research, 36, 16-47.

Rohner, R. P., \& Cournoyer, D. E. (1994). Universals in youths' perceptions of parental acceptance and rejection: Evidence from factor analyses within eight sociocultural groups worldwide. Cross-Cultural Research, 28, 371-383.

Rohner, R. P., \& Khaleque, A. (2002). Parental acceptance-rejection and life-span development: A universal perspective. In W. J. Lonner, D. L. Dinnell, S. A. Hayes, \& D. N. Sattler (Eds.), Online readings in psychology and culture (Unit 11, Chapter 4), Center for Cross-Cultural Research, Western Washington University, Bellingham, WA. Retrieved July 9, 2009, from http://www.ac.wwu.edu/ culture/Rohner_Khaleque.htm.

Rohner, R. P., \& Khaleque, A. (2005a). Parental Acceptance-Rejection Questionnaire (PARQ): Test manual. In R.P. Rohner \& A. Khaleque (Eds.), Handbook for the study of parental acceptance and rejection (4th ed., pp. 43-106). Storrs, CT: Rohner Research Publications.

Rohner, R. P., \& Khaleque, A. (2005b). Personality Assessment Questionnaire (PAR): Test manual. In R. P. Rohner \& A. Khaleque (Eds.), Handbook for the study of parental acceptance and rejection (4th ed., Pp. 187-226). Storrs, CT: Rohner Research Publications.

Rohner, R. P., Khaleque, A., \& Cournoyer, D. E. (2009). Introduction to parental acceptance-rejection theory, methods, evidence, and implications. Unpublished manuscript, University of Connecticut, Center for the Study of Parental Acceptance and Rejection. Retrieved July 9, 2009, from http://www.cspar.uconn.edu. 
Rohner, R. P., Saavedra, J. M., \& Granum, E. O. (1978a). Development and validation of the parental acceptance and rejection questionnaire: Test manual. JSAS Catalog of Selected Documents in Psychology (Manuscript 1635), 8, 7-8.

Rohner, R. P., Saavedra, J. M., \& Granum, E. O. (1978b). Development and validation of the personality assessment questionnaire: Test manual. University of Connecticut, Center for the Study of Parental Acceptance and Rejection. Retrieved July 8, 2009, from http:// www.cspar.uconn.edu.

Salama, M. M. (1986). Perceived parental acceptance-rejection and personality dispositions among college students in Egypt. Egyptian Journal of Mental Health, 27, 145163.

Saroglou, V., \& Scariot, C. (2002). Humor styles questionnaire: Personality and educational correlates in Belgian high school and college students. European Journal of psychology, 16, 43-54.

Seligman, M.E.P. (2000). Positive psychology: An introduction. American Psychologist, 55, 5-14.

Swami, V. (2008). Translation and validation of the Malay Subjective Happiness Scale. Social Indicator Research, 88, 347-353.

Taher, D., Kazarian, S. S., \& Martin, R. A. (2008). Validation of the Arabic Humor Styles Questionnaire in a community sample of Lebanese in Lebanon. Journal of Cross-Cultural Psychology, 39, 552-564.

Vernon, P. A., Martin, R. A., Schermer, J. A., \& Mackie, A. (2008). A behavioral genetic investigation of humor styles and their correlations with the Big-5 personality dimensions. Personality and Individual Differences, 44, $1116-1125$.

Zidan, A. A. M. (nd). Students' perception of parental acceptance/rejection, and its relation to students' achievement motivation at Al-Jouf Teachers College. The Educational Journal. Retrieved July 6, 2009, from http://pubcouncil. kuniv .kw.joe/english.

\section{Acknowledgments}

We thank Professor Ronald P. Rohner for making the Arabic version of the Parental Acceptance-Rejection Questionnaire available for our use. 


\section{About the authors:}

Shahe S. Kazarian has been a professor of clinical psychology at the American University of Beirut since 2001. His research interests focus on cultural clinical psychology, cultural health psychology, family functioning, and the psychology of humor.

Address for correspondence: Shahe S. Kazarian, Department of Social and Behavioral Sciences, American University of Beirut, 3 Dag Hammarskjold Plaza, $8^{\text {th }}$ Floor, NYC, NY 10017

E-mail: sk29@aub.edu.lb

Lamia Moghnie completed her master's degree in psychology at the American University of Beirut in August 2009. She also completed a master's degree in social science at the University of Chicago in 2006. She is currently a PhD student in Social Work and Anthropology at the University of Michigan and her research interests focus on the study of trauma as an object of diagnosis that (re) defines grief, loss and suffering in Lebanon.

Rod A. Martin has been a professor of clinical psychology at the University of Western Ontario since 1984. His research interests focus on the psychology of humor, particularly the role of humor in mental health and interpersonal relationships. He is on the editorial board of Humor: International Jo urnal of Humor Research. 